\title{
Challenges of an Internet of Things-Based Health Monitoring System
}

\author{
${ }^{1}$ D. Arun Shunmugam, ${ }^{2}$ Dr. K.Ruba Soundar, ${ }^{3}$ M. Desiya Narayan \\ ${ }^{1}$ Assistant Professor, Department of Computer Science and Engineering, P.S.R. Engineering College, \\ Sivakasi, Tamil Nadu, India \\ ${ }^{2}$ Professor and Head, Department of Computer Science and Engineering, P.S.R. Engineering \\ College, Sivakasi, Tamil Nadu, India \\ ${ }^{3}$ PG Research Scholar, Department of Computer Science and Engineering, P.S.R. Engineering \\ College, Sivakasi, Tamil Nadu, India
}

\begin{abstract}
The Internet of Things (IoT) is a cutting edge and quickly advancing innovation wherein everything (brilliant items and keen gadgets) is connected to the web for effective correspondence between them. The web of things is an impetus for medical care and assumes a basic part in an assortment of medical services following applications. By gathering internal heat level, circulatory strain, and sugar levels, organized sensors gadgets, regardless of whether worn on the body or installed in living conditions; permit the assortment of rich information to decide a patient's physical and psychological well-being condition. The troublesome errand in the Internet of things is conveying the gathered information to the specialist, settling on right choices dependent on the information gathered, and advising the patient. The creator of this paper centers around an investigation of IoT-based medical care frameworks, just as promising circumstances and difficulties for IoT-based patient wellbeing checking frameworks.
\end{abstract}

Keywords - Body Area Network, Healthcare, Internet of Things, Wireless Sensor Network

\section{Introduction}

Life in a period where each actual substance can be connected to another to share data. Numerous things or articles around us will share data naturally because of improved remote innovation like 6LoWPAN, Wi$\mathrm{Fi}$, Bluetooth, and ZigBee. The Internet of Things (IoT) is an organization of things or articles that are connected to one another through the Internet, a neighborhood, or remote sensor organizations. The Internet of Things is comprised of two words: the first is the Internet, and the second is Things. The Internet is an overall organization of organizations connected by standard conventions. Any Physical Object that might be associated with availability is alluded to as a "thing".

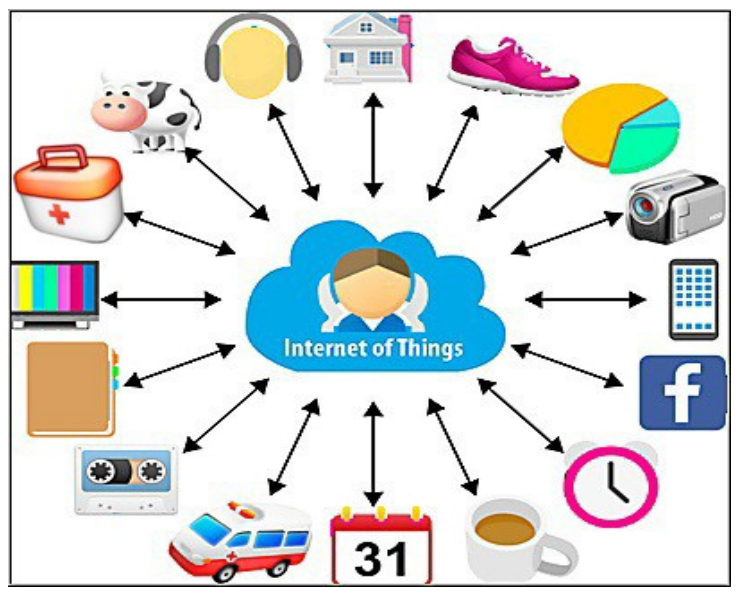

Fig 1. Internet Of Things ( IoT)
IoT utilizes an assortment of advancements, including RFID labels, sensors, actuators, and cell phone and distributed computing support, among others. We can interface anything, access any help, and valuable data about any article utilizing the Internet of Things [1].

\section{Economic Impact of IoT}

Many new technologies, such as microelectromechanical systems (MEMS), wireless sensor technologies, and the internet, have accelerated the growth of IoT. We have sensor devices for sale at a reasonable price. According to industry experts, about 25 billion IoT devices will be deployed by 2020 , and the IoT market will be worth around 2.1 trillion dollars by 2025[2]. According to marketresearch.com, the internet of things market in health care will hit $\$ 117$ billion by 2020[3.]. In June 2015, the McKinsey Global Institute published a study titled "the internet of things: mapping the potential beyond the hype," which forecast that the IoT market would grow to \$11.1 trillion per year by 2025[4]. According to Gartner, by 2020, up to 26 billion IoT devices will be connected to the Internet. According to Intechno Consulting, the IoT application market will generate up to 180 billion Euros globally [6]. 


\section{IoT Application Areas}

Close to Field Communication (NFC), Radio Frequency Identification (RFID), Machine-toMachine Communication (M2M), and Vehicle-toVehicle Communication (V2V) are the innovations that are permitting the Internet of Things to create at a fast speed. By 2020, it is normal that more than 50 billion IoT gadgets will be associated with the web. It will modify human existence, working styles, amusement alternatives, and considerably more [7]. The Internet of Things (IoT) has a wide scope of utilizations. The extension and space of these applications are extending constantly.

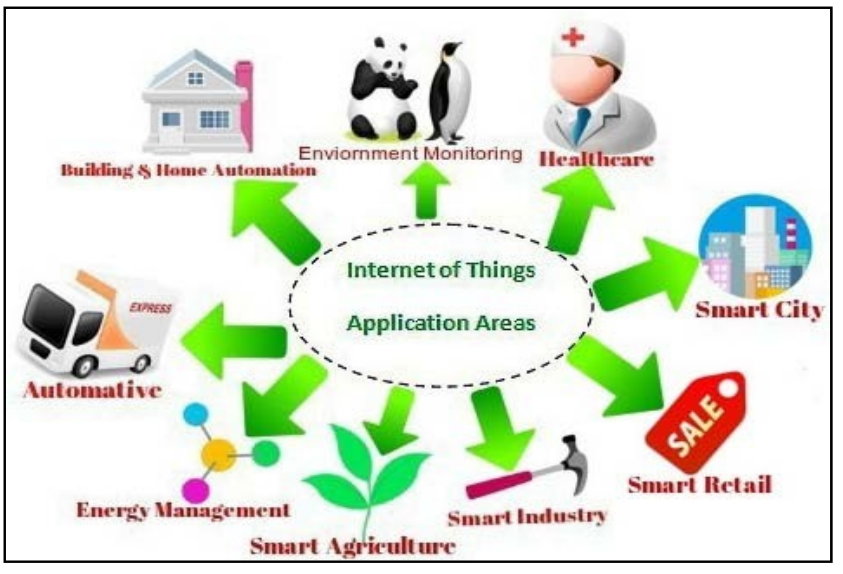

Fig 2. IoT Application Areas

There are a few IoT executions, including the accompanying:

- Smart Cities

- Building and Home Automation

- Environmental Monitoring

- Automotive Industry

- Smart Retail

- Smart Agriculture

- Smart Industry

- Energy Management

- Healthcare Monitoring

\section{Internet of Things and Healthcare Monitoring}

One of the businesses where IoT innovation is flourishing is medical care. As per Forbes magazine, by 2020, the IoT market in medical care will be worth more than $\$ 117$ billion [3]. As per a P\&S Market Research study, the medical care Internet of Things industry will extend at a build yearly development rate (CAGR) of 37.6 percent somewhere in the range of 2015 and 2020. IoT can possibly diminish the requirement for human-helped medical services. Patient wellbeing data, for example, pulse, internal heat level, and breathing example is recorded by IoT wearable gadgets (clinical sensors). This data will be shipped off the proper medical clinic or guardian for additional activity.

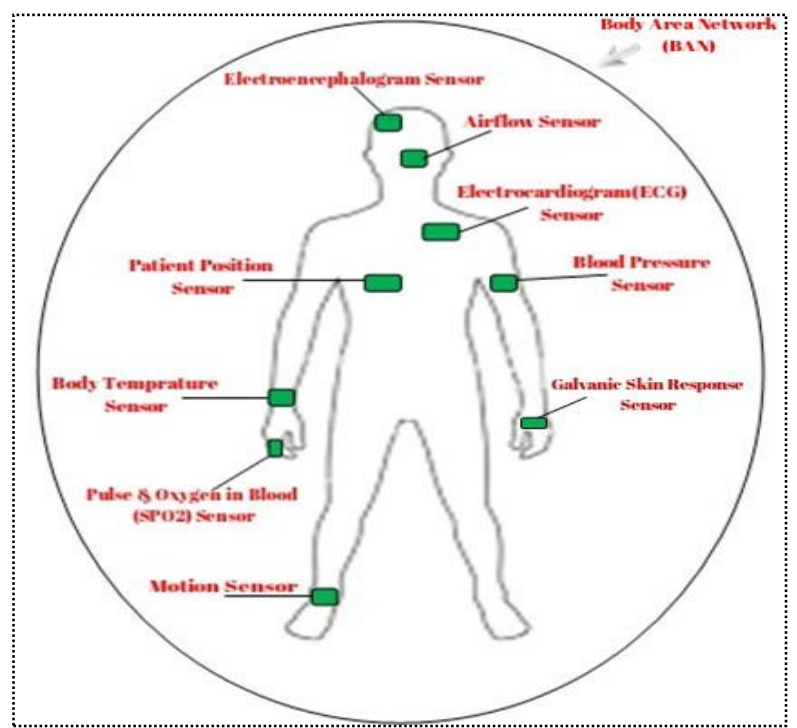

Fig 3. Body Area Networks

\section{IoT-Based Healthcare Monitoring uses Medical Sensors and Wearables}

It is presently conceivable to follow ailments distantly utilizing wearable gadgets because of improved remote network, clinical sensor, and information examination methods. Sensors and wearable gadgets can be incorporated into an assortment of embellishments, including dress, wristbands, eyeglasses, socks, covers, and shoes, just as different gadgets like cell phones, earphones, and wristwatches.

There are two sorts of clinical sensors: on-body contact sensors and fringe non-contact sensors. Body contact sensors are additionally isolated into two gatherings:

- Therapeutic sensors for medication (drug conveyance patches), unwinding (constant relief from discomfort), and crisis (ECG, EMG, EEG), compound (sweat, glucose, spit), visual (Oximetry, tissue properties), and crisis (ECG, EMG, EEG) (defibrillator).

- Non-contact sensors on the fringe are characterized into three sorts:

- Calculating movement (active work, carbohydrate content), and position to screen wellness and wellbeing (GPS, indoor limitation)

- Activity (fall, rest, work out), Emotion (uneasiness, strain, sadness), and Diet 
(calorie admission, dietary patterns) social control

- Rehabilitation by discourse (language advancement) and photography (daze technology) [8].

- Clinical sensors and wearable frameworks can be applied to the accompanying medical care applications [9].

- Hospital crucial sign following

- At-home and versatile maturing

- Motor and tactile misfortune help

- Large-scale in-field clinical and conduct research

\section{Health-Monitoring IoT Applications}

IoT may have an assortment of employments in the clinical field, including improving personal satisfaction, saving lives, and bringing down medical services costs. The clinical business can upgrade its capacity to lessen human mistake, work on the treatment cycle, and improve the personal satisfaction for parental figures and patients by carrying out IoT-based advancements. Specialists may utilize an IoT-based observing gadget to help in mind and anticipate a side effect prior to starting a conclusion. In health-related crisis cases, for example, when an old patient falls or shows dubious conduct in an emergency unit, checking framework may sound a caution (ICU). There are a few IoTbased medical services use cases and applications, including the following [10]:

- Health observation

- Personal wellness reconnaissance

- Chronic illness reconnaissance

- Safety reconnaissance

- Medication reconnaissance

- Real-Time Location Tracking

- Home Rehabilitation

\section{Health Surveillance}

Clinical screens and wearable gadgets can record fundamental finishes paperwork for use in wellbeing following and individual work out schedules. Sensors can be utilized to control pulse, blood glucose, weight, ECG, pulse, and internal heat level in youngsters and the older [11]

\section{Personal Fitness Surveillance}

This sort of sensor application is intended for individuals who need to keep up their wellness and wellbeing. Sensors can likewise be utilized to screen individual wellbeing and advancement. Numerous rules can be followed and recorded to assess an individual's prosperity and exercise schedule. Weight sensors, movement screen sensors, for example, strolling time counters, step counters, pace counters, calorie counters, and pulse and circulatory strain sensors are completely utilized here.

\section{Chronic Diseases Monitoring}

Ongoing infections like malignancy, diabetes, asthma, coronary illness, rest problems, and joint pain influence a great many individuals. Such a disease requires additional consideration. It required illness explicit dietary and treatment systems. Physiological sensors like ECG (electrocardiogram), EMG (electromyography), and EEG (electroencephalogram) joined with movement screen sensors, for example, step counter, pace counter, and calorie counter can be utilized to recognize signs and antagonistic changes in a patient's ailment early, taking into consideration brief clinical consideration.

\section{Safety Monitoring}

There are an assortment of sensors and wearable gadgets accessible to help with improving the medical care framework for the older and kids. Sensors for fall recognition, epileptic seizure's location, and coronary episode indication identification can be utilized for patient wellbeing observing. These sensors have a press button that conveys cautioning messages to guardians or relatives.

\section{Medication Management}

Rebelliousness with medication suggested by specialists is a typical human characteristic. This could jeopardize the patient's wellbeing just as result in monetary misfortune. For drug the board, an IoTbased astute bundling framework for medication boxes can be utilized. Controlled fixing is utilized in this bundling cycle, which depends on delaminating materials and constrained by remote communications [12].

\section{Home Rehabilitation}

The utilization of the Internet of Things in medical care can possibly improve recuperation. Detecting advancements dependent on the Internet of Things, joined with Virtual Reality conditions and expanded criticism frameworks, can be utilized to make a locally established recuperation framework for the older. Distant meetings are likewise conceivable with IoT-based technologies [13].

\section{Real-Time Location Tracking}

Web of Things (IoT) It is feasible to screen patients and the hardware utilized in medicines. Medical services suppliers can screen constant area, alloted doctor, and therapy progress utilizing RFID labels. 
Defibrillators, ECG machines, spirometry, and nebulizers, among other clinical gadgets, can be marked with sensors and checked effectively utilizing IoT [14].

\section{IoT-Based Healthcare Monitoring Technologies}

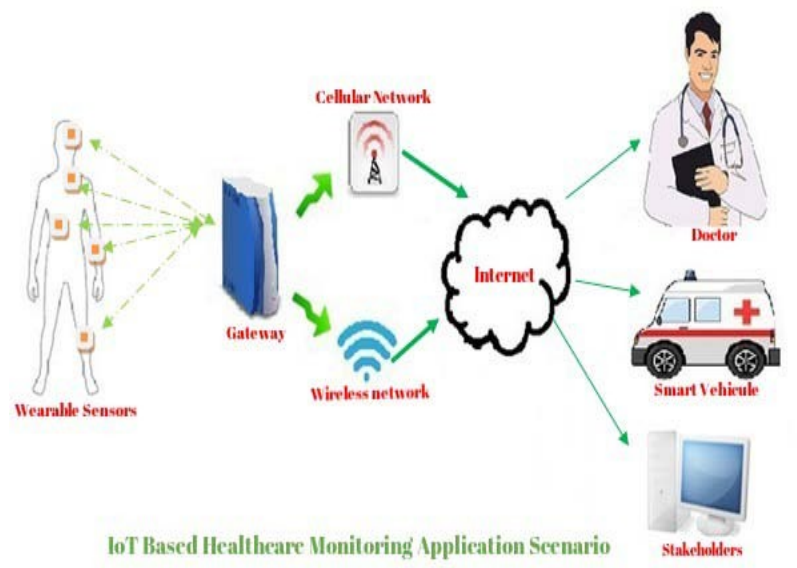

Fig 4. IoT in Healthcare

IoT is another innovation that interfaces all (living and nonliving) things to the Internet for information sharing and controller. IoT executions are made conceivable by joining heterogeneous innovations. RFID innovation, Sensor Networks, Wireless Communication Technology, and Embedded System Technology are totally utilized in IoT in healthcare[14]. The sensor gathers actual wellbeing information, which is then shipped off the middleware/entryway. The passage can oblige a few innovations and sensors simultaneously. It investigations and totals this wellbeing information prior to sending it to the web, where it is utilized by related medical care specialist organizations and investors to decide.

\section{Healthcare Benefits of IoT Use}

\section{Lower Hospital Costs}

Web of Things-based medical care offers constant patient following 24 hours per day, seven days per week. It would without a doubt eliminate pointless medical clinic visits just as transportation costs. Patients can get specialist exhortation at home through online video web based, and just in hazardous circumstances should they go to a medical clinic. IoT-based medical care following will bring down protection rates and empower patients to require less days off. Decrease in human mistake: In the field of IoT-based medical services following, Physical wellbeing information, for example, pulse and sugar levels, is acquired with changing precision by sensors, and ensuing choices are made utilizing enormous information investigation. It helps in the decrease of human mistakes.

\section{Dismantle Geographic Barriers}

Since doctors and patients are connected universally by means of the web, any tolerant can look for clinical guidance from anyplace on the planet.

\section{Paperwork and Documents to the Bare Minimum}

The Internet of Things Green innovation is upheld by web medical care following, which decreases desk work and detailing.

\section{Early Detection of Chronic Disorders}

It is feasible to anticipate persistent problems in their beginning phases and treat them before they become hopeless utilizing Big information examination and information mining methods on actual wellbeing data given by clinical sensors [16].

\section{Enhanced Drug Management}

It is a huge test to make and oversee drugs for the wellbeing business. By utilizing RFID (Radiorecurrence Identification) innovation in drug production network the board this industry can have better medication the executives for makers, providers, and customers moreover. It will lessen misfortune on account of the cheat, lost, and miss the executives of medications.

\section{Immediate Medical Attention}

IoT-based clinical gadgets can alert medical services suppliers or relatives if there should be an occurrence of a health-related crisis like an ascent in pulse or a fall of a senior relative.

\section{Better Outcomes of Treatment}

Day in and day out wellbeing observation and proofbased consideration choices will help in the ideal fix of sicknesses. Accordingly, treatment results will improve.

\section{Difficulties and Problems}

Any new innovation faces a few challenges. Medical services checking dependent on IoT has its own arrangement of bottlenecks and hindrances. Coming up next are a couple of them:

\section{Security and Privacy Issues}

Private medical care data is caught by medical care gadgets and programming, which are connected to the web for access whenever and from any spot. Subsequently, it can pull in programmers hoping to 
take individual data. After the patient has given authorization, private wellbeing records should be utilized. Robbery of a PC or clinical gadget for medical care, as indicated by true penetrate reports from 2009 to mid-April 2013, represents 51\% of by and large security risks [15].

- Health-care gadget actual assurance

- Ensuring safe information correspondence steering

- Ensuring information straightforwardness in a distributed computing climate

- Maximum security for minimal measure of assets

Patient wellbeing data is gotten from different clinical sensors and wearable gadgets in IoT-based medical care. For information assortment, clinical gadgets should connection to different gadgets and various clients. There are a great many merchants who produce items without holding fast to any uniform standards or guidelines for between gadget correspondence interfaces and conventions. Thus, the information gathered by these gadgets are not accessible to different gadgets. Interoperability issues emerge subsequently. Information from various IoT gadgets can get secured every framework because of an absence of interoperability, losing its likely worth, and expanding framework incorporation costs.

\section{Device Designing Issue}

In medical care, IoT gadgets are little sensors with low handling power processors, restricted extra room, and restricted battery power. IoT gadgets are frequently portable and connected to the web. Wearable frameworks should have the option to speak with each other. various organizations that give parental figures clinical subtleties It's as yet an examination challenge to make IoT gadgets with really preparing power, extra room, battery life, and versatility well-disposed assurance.

\section{Scalability}

In the coming days, a superior clinical framework would be accessible available. Countless IoT gadgets would be connected to the organization, producing gigantic measures of wellbeing information. The measure of information that should be put away and prepared will develop at a quick rate. Medical care will confront a major information challenge subsequently. Versatility is needed for the framework that stores and examinations information from IoT gadgets. Large information investigation and distributed storage are expected to improve treatment plans utilizing information acquired from associated IoT gadgets. As the quantity of IoT gadgets develops, creating data and perceptions from this information turns out to be more troublesome.

\section{Trust}

Clinical gadgets deliver and appropriate information that is helpless against digital assaults. While information may give off an impression of being correct, it very well might be undermined or adulterated by infection malware during transmission. Programmers can utilize this data to harm people since guardians settle on choices and treatment plans dependent on the information created by these sensors. Therefore, this spoiled information will prompt critical choices. How might we confide in a therapy subject to information from clinical sensors? In IoT-based wellbeing following, this is a critical obstruction.

\section{Conclusion}

The Internet of Things is just in its beginning phases; however, it can possibly hugely affect human medical services and related business sectors. It is feasible to screen people and different items because of rapid web network and progressed sensor innovation. Numerous specialized strategies to change the medical services framework have started to be found by scientists. This paper digs further into the Internet of Things (IoT)- based medical services applications, empowering innovation, and existing medical services issues and issues.

\section{References}

[1] "Who Needs the Internet of Things?" says Eric Brown. Linux.com is a site devoted to Linux. Wednesday, September thirteenth, 2016

[2] Ramakrishnan Iyer and Radharaman Mishra, "Building Intelligent Internet of Things Applications with Microsoft Stream Insight", IGATE Global Solution, April 2014, pp 1-7.

[3] T. J. McCue, "Web of Things in Healthcare: \$117 Billion Opportunity by 2020," [Online]. http://www.forbes.com/locales/tjmccue/2015/04/02/1 17-billion-market-for-web of-things-in-medical care by-2020/\#58d793712471

[4] "The Internet of Things: Mapping the Value Beyond the Hype," by James Manyika and Michael Chui, June 2015.

[5] James Manyika, "Organizations that move quickly on the Internet of Things will flourish now regardless of whether the economy needs to stand by," https://www.linkedin.com/beat/organizations movingquick web things-can-profit now-manyika

[6] Cyril Cecchinel, Matthieu Jimenez, Sébastien Mosser, and Michel Riveill, "An Architecture to Help Big Data Collection in the Internet of Things," IEEE tenth World Congress on Services 2014. 
[7] "The Internet of Things," by D. Evans How Will the Internet Change in the Future? No. April 2011, "The Internet of Things: How the Next Evolution of the Internet Is Changing Everything."

[8] Kunal Mankodiya, Shivayogi Hiremath, Geng Yang "Idea, Architectural Components, and Promises for Person-Centered Healthcare: Wearable Internet of Things" 2014, ICST

[9] "Wireless Sensor Networks for Healthcare," Jeong Gil Ko, Chenyang Lu, and Mani B. Srivastava. IEEE 2010 (IEEE)

[10]3rd International Conference on Computer Science and Network Technology, Xu Xingmei, Zhou Jing, and Wang He, "Exploration on the Basic Characteristics, Key Technologies, Network Architecture, and Security Problems of the Internet of Things"

\section{Author's Profile}

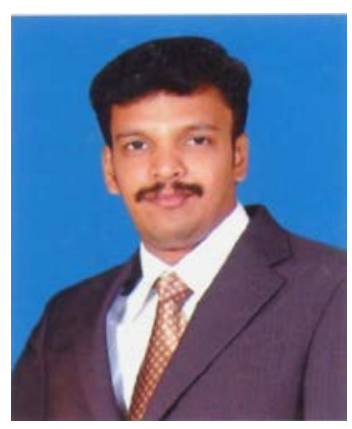

D. Arun Shunmugam working as a Assistant Professor, P.S.R. Engineering College, Sivakasi. I have 14 years of experience in Teaching field. I am doing my research in the area of Natural Language Processing from Anna University, Chennai.

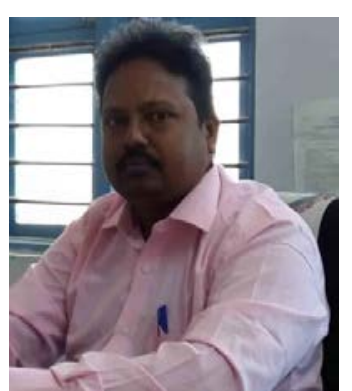

Ruba Soundar Kathavarayan received the A.M.I.E., degree in Computer Science and Engineering from The Institution of Engineers (India) in 2000. He received the M.E., and Ph.D., degrees in Computer Science and Engineering from Anna University, Chennai in the year 2004 and 2010 respectively. Currently he is a Professor in Computer Science and Engineering Department of P.S.R. Engineering College, Sivakasi, Tamil Nadu, India. He has authored / coauthored over 120 research articles in various Journals and Conferences in the areas of Cloud Computing, Image Processing, Wireless and Wired Networking.

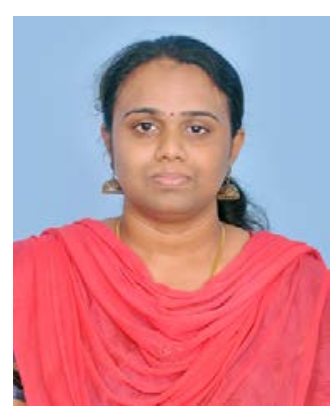

Deshiya Narayan. M completed my graduation in $\mathrm{P}$. S. E Engineering College(2015-2019). I'm doing my master of Engineering in computer science and engineering streamin P. S. R Engineering college (2109-2021). 